AUTHOR'S ABSTRACT OF THIS PAPER ISSUED BY

THE BIBLIOGRAPHIC SERVICE, NOVEMBER 17

\title{
A CASE OF A SYNDACTYLOUS CAT
}

GRACE P. HAYS

Reed College, Portland, Oregon

SIXTEEN FIGURES

An interesting case of syndactyly in a cat was brought to my attention by Prof. H. B. Torrey. The kitten was two or three months old when given to him. It lived for six weeks afterward. During this time it ate very little and this was obviously followed by much discomfort. Its death seemed to be due to starvation. At Professor Torrey's suggestion and under his direction I undertook to investigate the case. Very little could be learned of the ancestry. The mother was apparently normal, four years old. She had had but one kitten previous to this one and that was said to have been polydactylous with some deformity of the leg bones. The first kitten had been killed and nothing further could be learned about it. The father in the two cases is unknown.

The only other recorded case of a syndactylous cat which I have been able to find is that one noted by Freeland Howe, Jr. ('02). This cat had syndactylous hind paws each bearing two toes, and polydactylous fore paws, each with six toes. There was no description of the condition of the syndactylous feet and no account of the heredity. Very many cases of polydactyly occurring in cats have been recorded and a description of a case of syndactyly should be of value as affording a basis for comparison. In order to establish a working theory of the causes of such abnormalities in the extremities it is neccessary to have a record of the modifications of the abnormality which occur.

The significant features of this particular case of syndactyly are the very obvious tendency to a pronounced distal fusion, brought about by a partial obliteration of the central digits of 
the hands and feet, and the correspondence between the skeletal jrregularities and the irregularities of the muscles, pads, and claws.

Externally each of the two fore paws (fig. 1) shows two toes, and the skeleton reveals a pronounced symmetry. The condition of the skeleton of the hind feet is less regular. Externally the left hind paw has two toes and the right hind paw, three (fig. 2). These skeletal abnormalities may be seen by comparing the skiographs of the feet of this cat (figs. 3 and 5) with the skiographs of the feet of a normal cat (figs. 4 and 6 ).

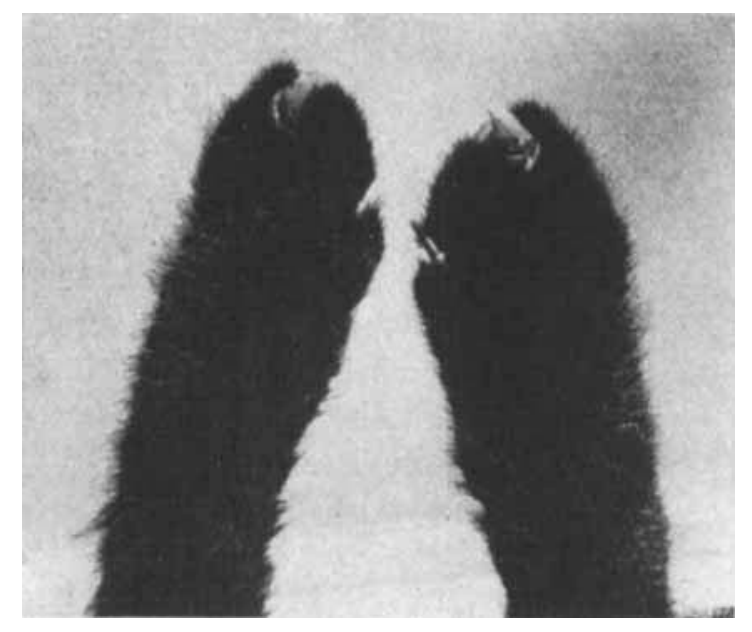

Fig. 1 Dorsal view of the fore feet of the abnormal cat.

In describing the bones reference will be made to the diagrams rather than to the skiographs. It must be remembered that the skiographs are shadow pictures and the prints therefore represent a mirror image of the actual condition. The foot on the right of the skiograph, then, must be compared with the diagram. of the left foot. Certain discrepancies may be noticed between the diagrams and the skiographs. It was impossible to flatten the foot absolutely under the X-ray and, as a result, the pictures are a little distorted. The diagrams are drawn with the aid of dividers from the dorsal side of the actual bones. 
Left fore foot. The bones of the first digit are normal throughout (fig. 7). The other digits are modified as follows. The diaphyses of the $2 \mathrm{~d}$ and 3rd metacarpals are distinct, and there is a bone which seems to have formed from the fused distal epiphyses of these bones. The distal boundary of this fused bone is partially obliterated in the skiograph. One bone represents the proximal phalanges of digits 2 and 3, and another, partially fused with this, the medial phalanges. The skiograph indicates a complete separation which is not found in the actual bones. There is no movable joint between the metacarpals

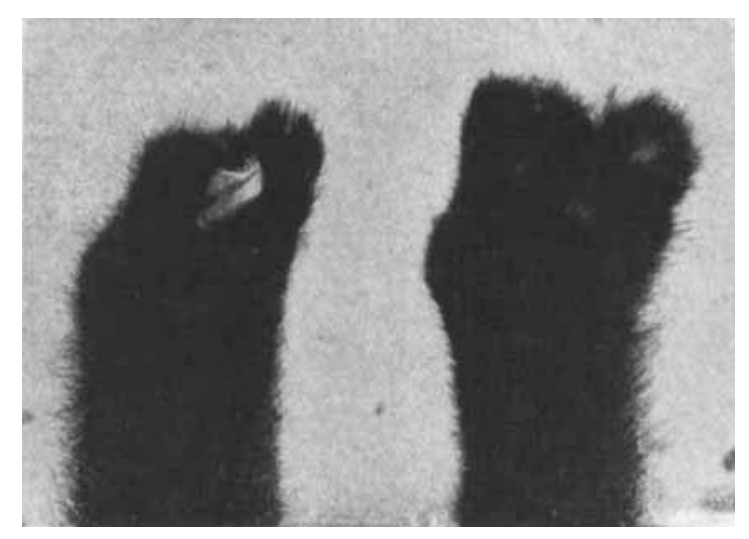

Fig. 2 Dorsal view of the hind feet of the abnormal cat.

and the proximal phalanx. Digits 4 and 5 indicate a similar pairing. The diaphyses of metacarpals 4 and 5 are separate. Metacarpal 5 has a distinct epiphysis. The epiphysis of metacarpal 4 has apparently fused with the bone which represents the 4th and 5th proximal phalanges. The proximal epiphysis of proximal phalanx 5 is distinct from the fused bone, although this does not show clearly in the skiograph. The medial phalanges of digits 4 and 5 are represented by one bone. Here, again, there is no movable joint between the metacarpals and the proximal phalanx, nor between the proximal and medial phalanges. There is but one distal phalanx, bearing one claw, 


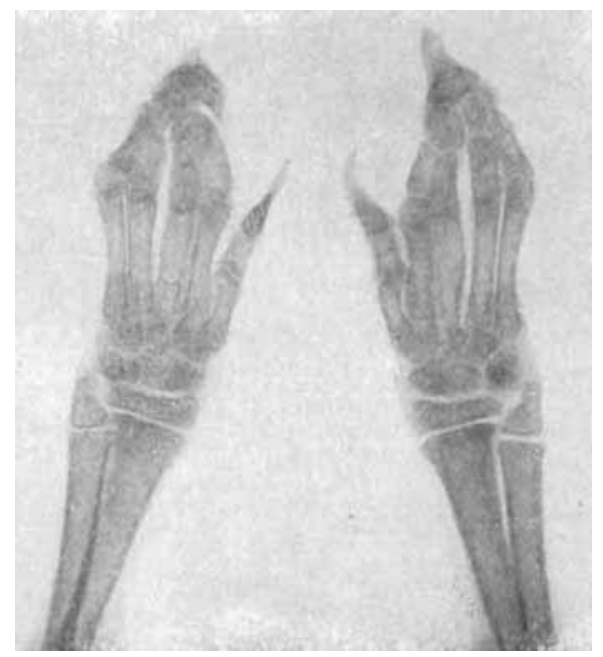

Fig. 3 Skiograph of the fore feet of the abnormal cat. The right foot is on the left. (The skiographs are shadow pictures and the prints therefore represent a mirror image of the actual condition.)

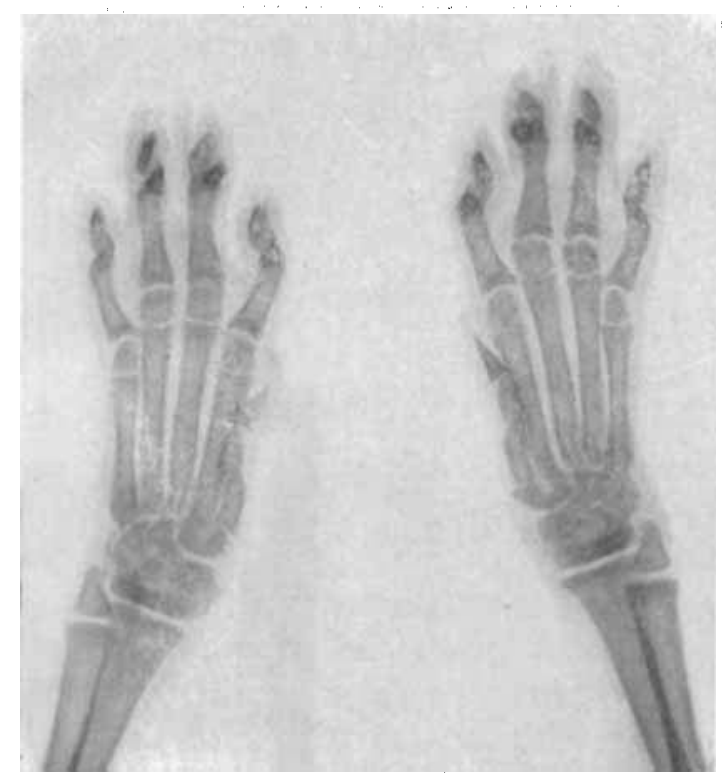

Fig. 4 Skiograph of the fore feet of a normal cat. The right foot is on the left. 


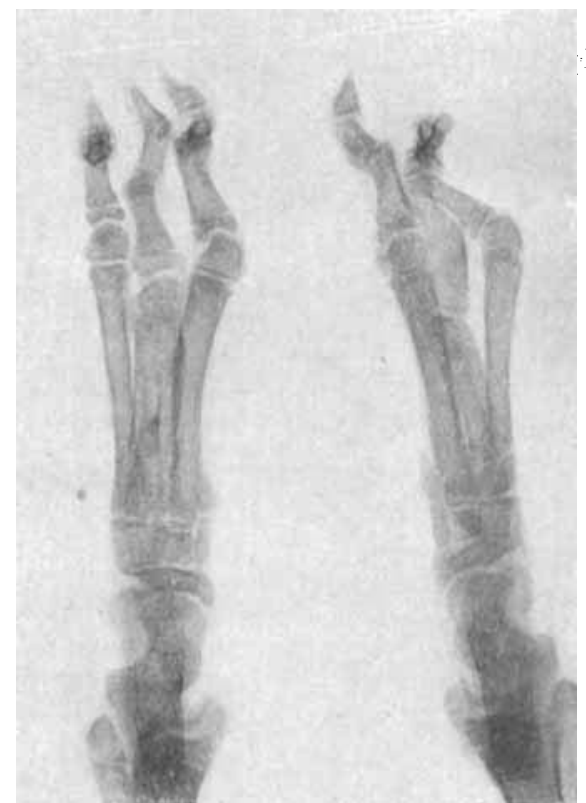

Fig. 5 Skiograph of the hind feet of the abnormal cat. The right foot is on the left.



Fig. 6 Skiograph of the hind feet of a normal cat. The right foot is on the left. 
to represent the four normally found. This distal phalanx articulates with medial phalanges $2-3$ and $4-5$.

Right fore foot. In the bones of this foot (fig. 8) the condition is so closely symmetrical to that described for the left that only the variations need be noted. The epiphysis of metacarpal 2 is distinct instead of being fused with the epiphysis of metacarpal 3. The epiphysis of metacarpal 3 is fused with the bone representing proximal phalanges 2 and 3 . The proximal epiphysis of metacarpal 4 is distinct. There are apparent dis-
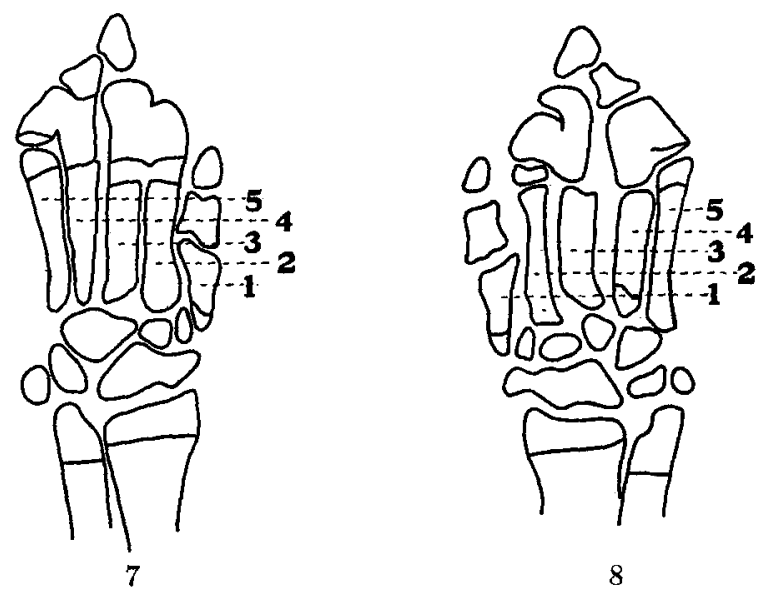

Fig. 7 Diagram of the bones of the left fore foot of the abnormal cat.

Fig. 8 Diagram of the bones of the right fore foot of the abnormal cat.

crepancies between the skiograph and the diagram in the relative dimensions of some of the bones of this foot. This is due to the fact that the foot was placed under the $\mathrm{X}$-ray in a plane not parallel to that of the photographic plate.

Left hind foot. The bones of digit 2 and the rudimentary bone representing the degenerate first digit are normal (fig. 9). The other digits are modified as follows. The diaphyses of metatarsals 3 and 4 have partially fused at the distal end. Their two epiphyses have fused. A single proximal and a single medial phalanx represent the proximal and medial phalanges of digits 3 and 4. Metatarsal 5 shows a distinct diaphysis and distal 
epiphysis, a proximal and a medial phalanx. Each of the proximal and medial phalanges shows a distinct proximal epiphysis. The distal phalanx representing digit $3-4$ and the distal phalanx of digit 5 have fused in a curious way. The bone thus formed articulates with the distal end of medial phalanx 3-4 and with the side of medial phalanx 5 . The claw borne by this distal phalanx shows the parts of two claws quite distinctly (fig. 2).

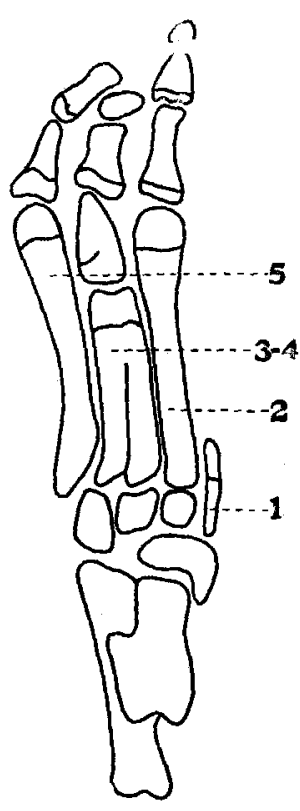

9

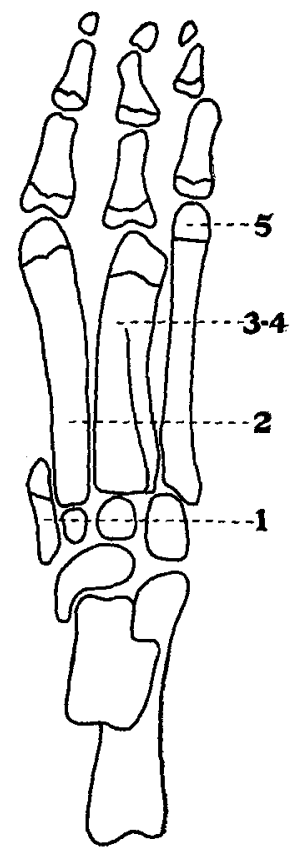

10)

Fig. 9 Diagram of the bones of the left hind foot of the abnormal cat.

Fig. 10 Diagram of the bones of the right hind foot of the abnormal cat.

The metatarsal bones of digit 3-4 are relatively short. As a result the distal end of the medial phalanx is approximately even with the distal end of the proximal phalanx of digit 5 . This discrepancy in length accounts for the peculiar articulation of the distal phalanx. The fusion of these distal phalanges has. resulted in a bending inward of the phalanges of digit 5 (fig. 5). 
Right hind foot. Digits 2 and 5 and the rudimentary bone representing digit 1 are normal (fig. 10). The diaphyses of the 3rd and 4th metatarsals have partially fused at the distal end; their epiphyses are completely fused, and there is a single representative of each of the phalanges. The diaphysis of digit $3-4$ is short, but not so pronouncedly as in the left pes. This shortness is compensated for in the length of the phalanges, so that the middle digit is not shorter than the lateral ones.

The wrist bones present few irregularities. In the left carpus the magnum and unciform have fused; otherwise the bones are normal. In the right carpus the magnum is small and does not articulate with the fourth metacarpal. The ankle bones are normal.

The tendency to obliteration in the central digits and to convergence to a point distally is such as one would expect to find resulting from a pressure directed proximad and effective along the distal margin and to some extent along the lateral margins. This tendency shows itself in two ways. In the fore paws the 3rd and 4th digits are shorter instead of longer than the others and the epiphyses are less frequently distinct from their diaphyses In the hind paws there is a conspicuous shortening of the partially fused 3rd and 4 th metatarsals, and the phalanges of these have completely merged their identity. As a result of this convergence and tendency to a dropping out or shortening of the central elements, the hands do not spread out in a distal direction as is normal, but have the shape, in general, of an inverted $V$. This is not so conspicuous in the feet, but in both cases there may be said to be a greater tendency to fusion distally than proximally.

There is in the fore feet also, a tendency to a synarthrosis, rather than a diarthrosis at the finger joints, that is, some of the joints which are normally articulate are non-articulate in this cat. Apparently at these joints the joint cavities failed to form, possibly because of some pressure at the distal margin, and the intervening tissue developed into a cartilaginous mass.

The non-articulate structure of the feet was partially responsible for the awkward gait of the kitten which at once 
attracted attention. In addition to inability to flex the bones normally, there was an abnormal muscular condition. The emaciated condition of the cat made dissection of the muscles of the feet difficult, but certain facts were discernable. There were no flexor muscles for the claws so that they could not be retracted. The plan of the musculature followed the skeletal plan, in general. Where the bones of the two digits were represented by single bones, the muscles were correspondingly represented by single muscles in the main. Certain muscles seemed to be lacking entirely and all were degenerate.

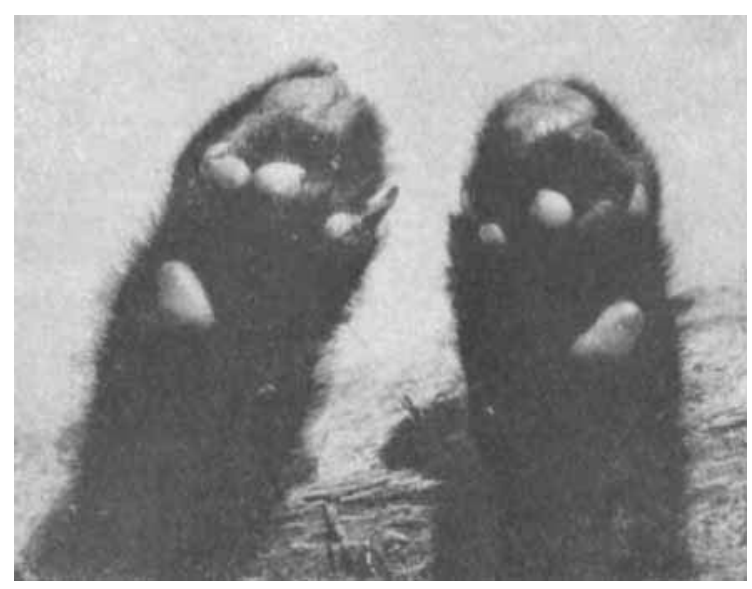

Fig. 11 Ventral view of the fore fect of the abnormal cat showing the abnormal condition of the pads.

Even the pads show an irregular condition (figs. 11 and 12) and those of the fore feet are again the more abnormal. A comparison of the diagrams of the normal (figs. 14 and 16) and abnormal (figs. 13 and 15) pads makes the discrepancies at once obvious. The pad usually found on the pollex of the first digit of the fore foot is found on the abnormal paws (figs. 13 and 14, a). The four pads normally found on the other digits (fig. 14, b, $c, d, e)$ are represented on the paws of the abnormal cat by one large pad (fig. 13, $b$ ). The three lobed metacarpal pads normally found (fig. 14, $f$ ) are represented in the abnormal condition by 
two separate pads on the right paw (fig. $13, f, f^{1}$ ) and by three separate pads on the left paw (fig. 13, $f, f^{1}, f^{2}$ ). The proximal pads (figs. 13 and $14, g$ ) seem to be similar in the two cases although the shorter hand in the case of the abnormal cat brings them in that case nearer the digital pads.

On the right hind paw there are three digital pads (fig. 15, $b, c, e$ ) corresponding to the normal four (fig. 16, $b, c, d, e$ ). On

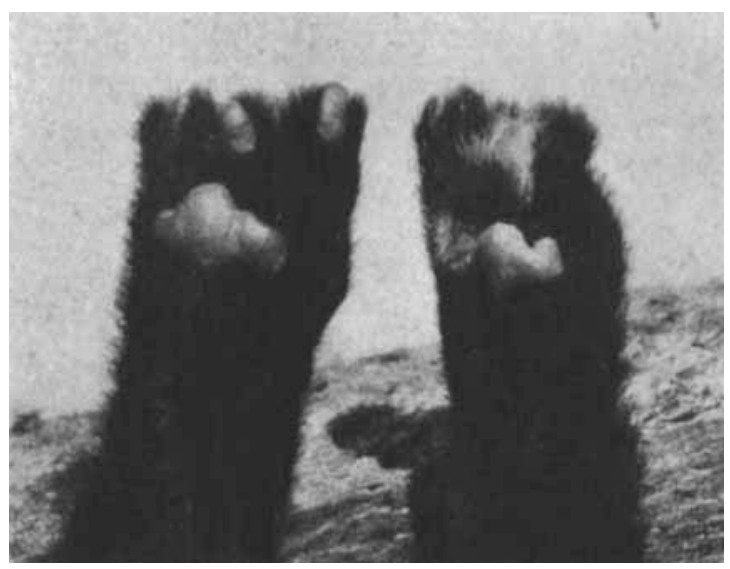

Fig. 12 Ventral vicw of the hind feet of the abnormal cat showing the pads.

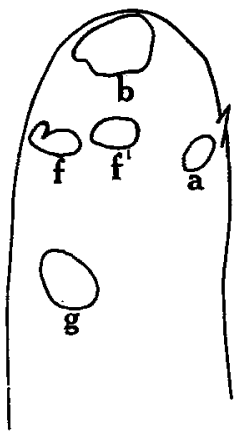

13

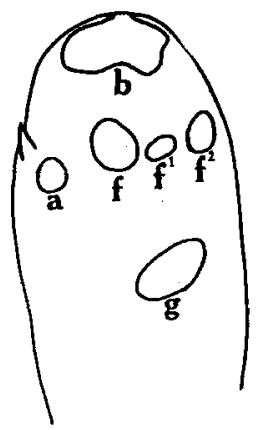

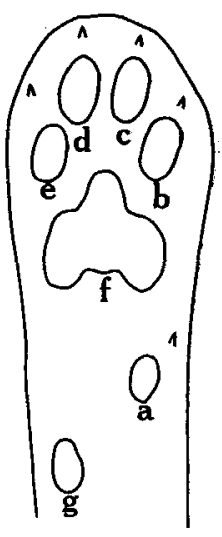

14

Fig. 13 Diagram of the pads on the fore feet of the abnormal cat. $a, b$, digital pads, $f, f^{1}, f^{2}$, metacarpal pads, $g$, proximal pads.

Iig. 14 Diagram of the pads on the right fore foot of a normal cat. $a, b, c, d$, $e$, digital pads, $f$, metacarpal pad, $g$, proximal pad. 
the left foot there are but two digital pads, (fig. 15, b,c). The metatarsal pads in each case are three lobed (fig. 15, f) as is normal (fig. 16, $f$ ).

There exists then, a definite correlation between the variations of the bones, muscles, and pads.

In this cat a condition of malnutrition existed in the postfoetal life. The difficulty was not so much one of non-assimilation as of actual starvation. The eat ate very little after it was brought into the laboratory and this was obviously followed by

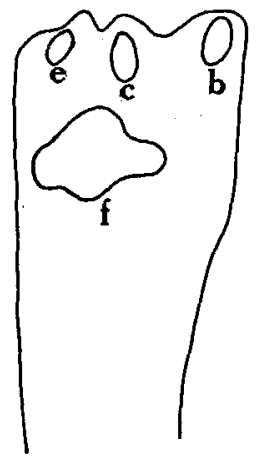

15

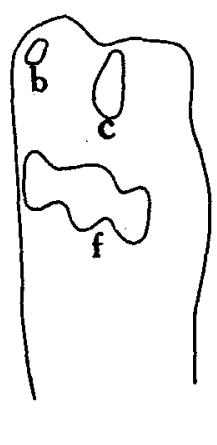

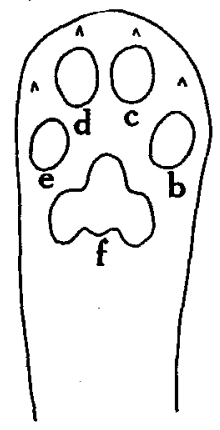

16

Fig. 15 Diagram of the pads on the hind feet of the abnormal cat. $b, c, e$, digital pads, $f$, metatarsal pads.

Fig. 16 Diagram of the pads on the right hind foot of a normal cat. $b, c, d, e$, digital pads, $f$, metatarsal pad.

much discomfort. At death the body was exceedingly emaciated. It was thought that there might be some clogging of the intestines but a dissection of the complete length of the alimentary canal revealed no mechanical obstruction. The stomach was quite empty and the intestines contained very little, a few wads of hair, three small thread worms, and several small quantities of waste material. Both the small and large intestine were greatly shrunken, although the lumen remained open. Owing to this, the diameter of the large intestine was less in proportion than is normal. Malnutrition undoubtedly caused the death of the kitten, but it is difficult to see how it could have factored 
in causing the defects which originated during foetal development. Both the physical deformities and the failure to eat, however, may have been the result of the same physiological disturbance.

Other than the variations noted the cat seemed to be quite normal. The thymus and pituitary body appeared normal. The thyreoid was a little small when compared to the thyreoid of a normal kitten of the same size. It was at first suspected that the upper leg bones were relatively short but a comparison of the radio-humeral and tibio-femoral indices with those of normal cats of the same age indicated no such unusual condition. The abnormalities under consideration were confined to the feet.

This case raises several questions. Is the deformity heritable? If so what is the heritable character? What are the relations of the various forms of abnormality occurring in the extremities? What are the causes? Are such deformities commonly associated with any other abnormal conditions?

Poulton ('83, '86), Bateson ('94) and Torrey ('02) have indicated that polydactyly is strongly hereditary in cats. Lewis ('09) and Broman ('04) establish the heritability of both polydactyly and syndactyly among human beings. Broman ('04, p. 62) says,

Viele, ja vielleicht die meisten angeborenen Missbildungen und Anomalien sind ausgesprochen erblich, und zwar sowohl HemmungsMissbildungen wie andere. Sehr erblich sind z. B. Hypospadie, Poly- und Syndactylie und die angeborene Hüftgelenksluxation (Hoffa, '04).

Both these authorities note the frequent association of polydactyly and syndactyly in the same individual. Broman ('04, p. 233) says,

"Die Syndactylie ist eine relativ gewöhnliche Missbildung, Sie kommt nicht selten entweder mit Perodactylie oder mit Polydactylie kombiniert vor."

In order to establish a working theory concerning the origin of polydactyly, where there is a greater than normal number of elements, and of syndactyly, where the number of elements is less than the normal number, it will be necessary to indicate the 
various types of malformation which occur in the extremities. Bateson ('94, p. 399) finds the cases of polydactyly more regular than those of syndactyly. Polydactyly, in its simplest form, may consist in the reduplication of a single phalanx. The intermediate step between it and the normal condition is the development of a third phalanx on the first digit or thumb. The reduplication may proceed so far as to include the metacarpal and metatarsal bones of several digits of hands and feet. Syndactyly seems to be more irregular in its manifestations. Cutaneous and fibrous forms occur in which only the fleshy parts of the extremities are affected. It may result from the omission of certain skeletal elements or from fusion of some of the bones. In such cases there is a corresponding syndactyly of the fleshy parts. The omission may occur in either marginal or central digits. The latter is less common and is called split hand or split foot (Broman, '04, pp. 651, 230-231). According to Lewis ('09, p. 7), split foot may be accompanied by polydactylous hands and split foot is never found unaccompanied by split foot. Defects in the wrist bones may occur. A single carpal or tarsal bone may completely fail or may fuse with a neighboring bone. In the case of an omission of one of the carpal or tarsal bones, there is usually a coincident defect of the bones either distal or proximal. (Broman, '04, p. 651).

In either polydactyly or syndactyly the abnormal condition is apt to be symmetrical (Broman, '04, p. 231) and to be more pronounced distally than proximally. This distal syndactyly was particularly conspicuous in the cat under discussion. Bateson finds this difficult to understand. He says ('94, p. 357).

The rule that in the lowest condition of syndactylism of the bones it is commonly at the periphery that the union is most complete is also difficult to understand in connexion with the fact that the division of digits in the lowest forms of polydactylism appears also in the peripheral phalanges.

Syndactyly and polydactyly, then, are both heritable and are frequently found in association. Each consists in an abnormal number of skeletal parts, the one in less and the other in greater number. The abnormality is usually more pronounced at the 
distal extremity. In analysing the possible causes of such conditions one must recognize that, although a given case may not be hereditary, it may be the result of conditions identical with those effective in hereditray cases. The difference would be that in the former case the effective factors must have arisen locally, while in the hereditary case such factors were carried in the germ cells.

The question of when the unknown factors causing polydactyly or syndactyly may be effective is important in determining what these factors may be and this can be answered only by a consideration of the course of normal development. Since no material on the cat is available, a review of the essential points of the development of the human arm will suffice. The lower extremities develop in a way essentially similar to the upper, and the facts regarding the cat may be considered different only in unessential details.

The skeleton of the hand first appears outlined in a bedplate of scleroblastema. In this, differentiation proceeds from base to tip, the humerus, radius and ulna being laid down in cartilage before the carpus and fingers. In the hand the metacarpal cartilage forms, then the carpal, and finally the cartilages of the phalanges; the cartilages of the distal phalanges are the last to appear. The first ossification occurs in the long bones. In the hand the distal phalanges ossify first, the metacarpals and proximal phalanges next, then the medial phalanges and finally the carpal bones (Bardeen '10, pp. 366-398).

As the various parts of the skeletal anlage become differentiated, the hand region assumes the form of a rounded pad. It is probably at this or an earlier stage that later poly-or syndactyly is determined. It is difficult to conceive how, after the cartilages had been laid down, certain elements could be eliminated in the case of syndactyly or how new cartilaginous elements could form in the case of polydactyly. It is quite conceivable, however, that, if this pad were prevented from rounding out to the normal width, differentiation might progress, with a crowding together of the elements such that some would fuse or, in extreme cases, even drop out. On the other hand if the pad were to widen to a 
greater degree than ordinarily, superfluous elements might be formed and a condition of polydactyly result. If the syndactyly or polydactyly occurred slightly later, after the anlagen of the digits were differentiated, it would have to result either from a fusion or from added separation. Syndactyly might be a result of some early fusion of distinct or partially distinct elements and polydactyly a result of a division of one or more of the digit anlagen. Broman ('04) supports both the idea that syndactyly arises early and the idea that it may be in some way a pressure effect. He says (p. 651).

In anderen Fällen ist die anscheinend kleinere Zahl der Finger (Zehen) auf mangelhafte Trennung (Syndactylie), der in normaler Zahl vorhanden Fingeranlagen zurückzuführen (fig. 199-202 s. 232). Die Skeletteile zweier mangelhaft getrennten Finger können entweder getrennt bleiben oder verschmelzen (Syndactylie ossea).

He does not explain what causes this defective separation nor does he give any evidence for assuming that syndactyly is brought about at this time. In regard to pressure he says ('04, p. 233),

"In vielen Fällen von Syndactylie scheint man berechtigt zu sein, die Missbildung auf frühzeitige Druckwirkung und Raumbeengung durch das Amnion zurückzuführen (Klaussner 1904)."

Neither of these views, which are as yet unsupported by adequate evidence, involves a consideration of the relation of syndactyly to polydactyly. There is no definite evidence pointing to distinct origins of these two conditions. On the contrary they are frequently found in close relation. This suggests that both may be due to the operation of a single fundamental factor.

It would be interesting to determine whether or not the heritable character of each of these abnormalities might be a tendency to instability in the formation of the extremity. If such were the case, the resulting polydactyly or syndactyly would depend upon individual conditions; if it were not, the two might be fundamentally different in origin. In the recorded strains of polydactylous cats there was no constant number of digits characteristic of a given family, but there seemed to be a tendency to increase the number of superfluous digits in the 
later generations. This probably points to some unstable factor in the underlying situation.

At present it seems to be simplest to think of either polydactyly or syndactyly as due to the introduction of some factor not normally present. Local conditions are never identically the same, and the variations in the abnormality may be the result of variations in the local conditions rather than the result of variations in the unusual factor. This idea can be expressed in abstract terms. The individual variable conditions governing the formation of a given extremity may be represented by $x$. A constant factor, $K$, may or may not be introduced. If introduced an abnormal condition is produced. $x+K$, then, may be said to represent the situation when syndactylous extremities are formed. If, however, the local conditions are sufficiently changed, and if to these changed local conditions the constant factor, $K$, is added, $X+K$ may produce polydactylous extremities. In a given individual the physiological condition of the upper extremities might be represented by $X$ and that of the lower extremities by $x$. If, then, the disturbing factor, $K$, were introduced such an individual would have polydactylous hands and syndactylous feet.

Such a factor might be either physiological or mechanical. If physiological, it must be a factor which occasionally affects only the upper or lower extremities. Three possible physiological factors at once occur to one, infection, malnutrition, and deficient blood supply. In practical effect the last two are the same, for the physiological condition would be the same whether the lack of material was occasioned by an actual lack of the blood supply, or by a lack of the essential constituents in the blood. The evidence taken from cases of rickets makes it clear that there is a distinct connection between nutrition and bone formation (Still, '14, p. 776). If the factor be mechanical it must tend to exert its effect symmetrically. The conception of pressure as the determining factor is not new. The amnion has been thought to be a possible source of pressure, causing both poly- and syndactyly. It has been suggested that polydactyly may result from pressure of amniotic threads on the distal 
margin of the hand plate (Broman, '04). To effect syndactyly the pressure would have to be exerted laterally. There are two chief difficulties in the way of such an explanation. If the amnion is the determining factor some evidence pointing to this should have come to light (Broman, '04). The chief difficulty, perhaps, is presented in the general symmetry of the abnormalities. It is difficult to see why the pressure, unless itself the result of some physiological condition, should be effective symmetrically. It is conceivable that lateral pressure might be effective in causing a change in the normal shape of the early limb pads, as was suggested above, but it is difficult to explain the source of the pressure.

Various other abnormalities are occasionally associated with polydactyly or syndactyly. No causal connection between these defects has been established and they are of no significance for the general condition under discussion. None of these defects were present in the kitten. Bateson ('94, p. 399) finds occasionally phocomely, cyclopia, double uterus, hare lip, defective dentition, defect of tibia, associated with polydactyly. Lewis ('09, p. 10) finds polydactyly occasionally in conjunction with hare lip and abnormalities of the limb bones. Bateson ('94, p. 399) finds syndactyly associated very often with general deformity and with many forms of arrested development. 


\section{BIBLIOGRAPHY}

Bardeen, Chas. R. 1901 The development of the limbs, body-wall and back. Am. Jour. Anat., vol. 1.

1905 Studies of development of the human skeleton. Am. Jour. Anat., vol. 4.

1910 Chapter XI in Keibel and Mall: Manual of human embryology, vol. 2.

BAteson, W. 1894 Materials for the study of variation treated with especial regard to discontinuity of species.

Broman, I. 1904 Normale und abnorme Entwickelung des Menschen.

Davison, Alvin 1911 Mammalian anatomy with especial reference to the cat.

Howe, Freeland, Jr. 1902 A case of abnormality in cat's paws. Am. Nat., vol. 36, pp. 511-526.

LEWIS, T. 1909 Sec. 2 and 3 in Treasury of human inheritance, I-II. Francis Galton Lab. for Nat. Eugenics.

Potuton, E. B. 1883, 1886 Observations on heredity in cats with an abnormal number of toes. Nature, vol. 29, pp. 20-21. Also Nature, vol. 35, pp. 38-41.

Prentiss, Caas. W. 1903 Polydactylism in man and the domestic animals with especial reference to digital variation in swine. Bul. Mus. Comp. Zool., vol. 40 , no. 6 .

ReIGHaRd and JeNNings 1901 The anatomy of the cat.

Still, Geo. F. 1914 Chap. 20 in Ostler and MeCrea: Modern medicine, vol. 2.

Stöhr, Philltp 1913 Textbook of histology (trans. Lewis).

Torrey, Harry Beal 1902 Prepotency in polydactylous cats. Science, N.S., vol. 16 , Oct. 3.

Winder, B. C. A. 1891 The occurrence of an additional phalanx in the human pollex. Jour. Anat. and Physiol., vol. 26. 